\title{
OPEN Urinary fatty acid binding protein 3 (UFABP3) is a potential biomarker for peripheral arterial disease
}

\author{
Abdelrahman Zamzam ${ }^{1}$, Muzammil H. Syed ${ }^{1}$, John Harlock ${ }^{2}$, John Eikelboom ${ }^{3,4}$, \\ Krishna K. Singh ${ }^{5}$, Rawand Abdin ${ }^{4} \&$ Mohammad Qadura ${ }^{1,6,7 凶}$
}

Plasma levels of fatty acid binding protein 3 (pFABP3) are elevated in patients with peripheral artery disease (PAD). Since the kidney filters FABP3 from circulation, we investigated whether urinary fatty acid binding protein 3 (UFABP3) is associated with PAD, and also explored its potential as a diagnostic biomarker for this disease state. A total of 130 patients were recruited from outpatient clinics at St. Michael's Hospital, comprising of 65 patients with PAD and 65 patients without PAD (non-PAD). Levels of UFABP3 normalized for urine creatinine (UFABP3/UCr) were 1.7-folds higher in patients with PAD [median (IOR) 4.41 (2.79-8.08)] compared with non-PAD controls [median (IOR) 2.49 (1.78-3.12), $\mathrm{p}$-value $=0.001]$. Subgroup analysis demonstrated no significant effect of cardiovascular risk factors (age, sex, hypertension, hypercholesteremia, diabetes and smoking) on uFABP3/UCr in both PAD and non-PAD patients. Spearmen correlation studies demonstrated a significant negative correlation between UFABP3/UCr and $A B I(p=-0.436 ; p$-value $=0.001)$. Regression analysis demonstrated that UFABP $3 / \mathrm{Cr}$ levels were associated with PAD independently of age, sex, hypercholesterolemia, smoking, prior history of coronary arterial disease and Estimated Glomerular Filtration rate (eGFR) [odds ratio: 2.34 (95\% confidence interval: 1.47-3.75) p-value < 0.001]. Lastly, receiver operator curve (ROC) analysis demonstrated unadjusted area under the curve (AUC) for UFABP3/Cr of 0.79 , which improved to 0.86 after adjusting for eGFR, age, hypercholesteremia, smoking and diabetes. In conclusion, our results demonstrate a strong association between UFABP $3 / \mathrm{Cr}$ and $\mathrm{PAD}$ and suggest the potential of UFABP3/Cr in identifying patients with PAD.

Peripheral arterial disease (PAD) is a chronic atherosclerotic condition that affects over 200 million people globally ${ }^{1-3}$. Despite its prevalence, a large proportion of patients with PAD receive delayed treatment, and consequently, face a high risk of lower-extremity amputations and mortality ${ }^{4-7}$.

Currently, the ankle brachial index (ABI) - the ratio of the brachial artery blood pressure to the ankle blood pressure-is the only validated screening tool for $\mathrm{PAD}^{8}$. However, the $\mathrm{ABI}$ has limitations; it is often erroneously interpreted in primary care, and can be unreliable in patients with diabetes ${ }^{9-11}$.

We have previously demonstrated an association between elevated plasma levels of fatty acid binding protein 3 (pFABP3) and $\mathrm{PAD}^{12}$. FABP3 is a small intracellular protein that is normally absent from plasma but is released into the circulation following myocardial or skeletal injury ${ }^{13,14}$.

Because the kidneys play an important role in clearing and filtering pFABP3 from circulation ${ }^{15-17}$, we have now investigated whether urine fatty acid binding protein (uFABP3) levels are elevated in patients with PAD and could potentially serve as a diagnostic biomarker for this disease state.

\section{Materials and methods}

Ethics approval. This study received approval from the research ethics board at St. Michael's Hospital-University of Toronto in Ontario, Canada. Informed verbal and written consent were obtained from all participants, and all methods were carried out in accordance with the relevant guidelines and regulations.

\footnotetext{
${ }^{1}$ Division of Vascular Surgery, St. Michael's Hospital, Toronto, ON M5B 1W8, Canada. 2Department of Surgery, Hamilton General Hospital, Hamilton, Canada. ${ }^{3}$ Population Health Research Institute, McMaster University, Hamilton, ON, Canada. 'Department of Medicine, McMaster University, Hamilton, ON L8S 4K1, Canada. ${ }^{5}$ Department of Medical Biophysics, Schulich School of Medicine and Dentistry, University of Western Ontario, London, ON N6A 5C1, Canada. ${ }^{6}$ Department of Surgery, University of Toronto, Toronto, ON M5S 1A1, Canada. ${ }^{7}$ Keenan Research Centre for Biomedical Science, Li Ka Shing Knowledge Institute, St. Michael's Hospital, Toronto, ON M5B 1W8, Canada. ${ }^{\boxplus}$ email: mohammad.qadura@utoronto.ca
} 
Patient recruitment. For this pilot study, the first encountered 65 PAD and 65 non-PAD patients presenting to ambulatory clinics at St. Michael's Hospital between April 2019 and March 2020 were recruited. As previously described ${ }^{18}$, patients with $\mathrm{PAD}$ were defined based on an $\mathrm{ABI}<0.9$, as well as abnormal distal pulses examination with or without claudication. A control group of patients without PAD were also recruited. The non-PAD control group were defined based on an $\mathrm{ABI} \geq 0.9$, presence of palpable distal pulses, and no clinical history of claudication. TBI (toe brachial index) measurements were performed where ABI values could not be calculated due to non-compressible tibial vessel. Patients with $\mathrm{TBI}<0.67$ were characterized as having PAD, whereas controls had a TBI of $\geq 0.67$.

Patients with a history of chronic kidney disease (stages 3, 4 and 5) or acute limb ischemia, and those with a history within the past 12 months of acute coronary syndrome (ACS), acute congestive heart failure (CHF), uncontrolled arrhythmia or elevated troponins were excluded.

Baseline measurements and sample collection. We recorded baseline demographics, history of cardiovascular diseases, cardiovascular risk factors, and smoking status ${ }^{19}$. Each patient also underwent lower limb arterial imaging with an ultrasound (US), ABI or toe brachial index (TBI). Patients were defined as having hypercholesterolemia if they had total cholesterol $>5.2 \mathrm{mmol} / \mathrm{L}$, triglyceride $>1.7 \mathrm{mmol} / \mathrm{L}$, or were taking lipid lowering therapy; as having hypertension if they had a systolic blood pressure $\geq 130 \mathrm{mmHg}$, diastolic pressure $\geq 80$ $\mathrm{mm} \mathrm{Hg}$, or were taking blood pressure lowering therapy; and as having diabetes if they had a glycosylated hemoglobin Alc $\geq 6.5 \%$ or were taking antidiabetic medication. Patients were defined as having renal disease if they had an estimated glomerular filtration rate of less than $60 \mathrm{~mL} / \mathrm{min} / 1.73 \mathrm{~m}^{2}$. The Estimated Glomerular Filtration rate (eGFR) was calculated for each patient as previously demonstrated by Levey et al. ${ }^{20}$.

Mid-stream urine samples were collected, aliquoted and stored at $-80{ }^{\circ} \mathrm{C}$ prior to analysis. Urine samples were thawed slowly on ice prior to analysis.

Urinary FABP3 multiplex assay. To determine the concentrations of FABP3 levels in urine (uFABP3), samples were examined in duplicate using MILLIPLEX MAP Human Cardiovascular Disease (CVD) Magnetic Bead Panel 1 (EMD-Millipore; Billerica, MA). To minimize any inter-assay variability, all analyses were carried out on the same day. Sample intra-assay and inter-assay CV were $<10 \%$. Prior to any sample analysis, Fluidics Verification and Calibration bead kits (Luminex Corp) were used to calibrate the MagPix analyzer (Luminex Corp; Austin, Texas). At least 50 beads for uFABP3 were acquired using Luminex xPonent software and analyzed using Milliplex Analyst software (v.5.1; EMD-Millipore).

Measurement of urinary creatinine and normalization of uFABP3. Urine creatinine (uCr) levels were measured at the Core laboratory at St. Michael's Hospital using the Beckman Coulter AU680 laboratory analyzer (Beckman Coulter; Pasadena, California). The uFABP3 concentration were normalized to uCr to adjust for urinary concentration errors and differences in hydration status, while relying on single-spot urine samples to achieve normalized $\mathrm{uFABP} 3 / \mathrm{uCr}(\mu \mathrm{g} / \mathrm{g})$.

Statistical methods. Data are presented as median and interquartile ranges (IQR). Normality was assessed using the Shapiro-Wilk test. Since the continuous variables were not normally distributed, the Mann-Whitney U or Kruskal-Wallis test was used to evaluate differences between groups. A post-hoc test was used for pairwise comparisons after multiple group testing. Fisher's exact test or chi-square test was used for categorical variables. In an attempt to compare uFABP3/uCr levels among PAD and non-PAD patients, patients with PAD where stratified based on ABI into mild (0.89-0.75), moderate (0.74-0.50) and severe PAD $(<0.50)$ groups as per the European Society for Vascular Medicine (ESVM) guidelines on peripheral arterial disease ${ }^{21}$. Logistic regression was used to evaluate the association between a one standard deviation increase in $\mathrm{uFABP} 3 / \mathrm{uCr}$ levels and diagnosis of PAD. Z-scores $\mathrm{uFABP} 3 / \mathrm{uCr}$ values were used for ease of odds ratio interpretation. Logistic regression models were used to examine the association between $\mathrm{uFABP} 3 / \mathrm{uCr}$ and other parameters with the development of PAD. Correlations between ABI (i.e. PAD severity) and uFABP3/uCr were analyzed using Spearman's correlation. Area under the curve (AUC) from receiver operating characteristic (ROC) analysis was used to determine the ability $\mathrm{uFABP} 3 / \mathrm{uCr}$ in distinguishing between patients with PAD and non-PAD patients. Moreover, to better understand the predictive capabilities of $\mathrm{uFABP} 3 / \mathrm{uCr}$, a ROC curve was estimated using probability estimates from a fitted model with PAD regressed on $\mathrm{uFABP} 3 / \mathrm{uCr}$ and age, hypercholesteremia, smoking and diabetes. All analyses were carried out at a 5\% two-sided significance level and carried out using SPSS software version 23 (SPSS Inc., Chicago, Illinois, USA).

\section{Results}

Cohort description and levels of UFABP3/UCr in controls and patients with PAD. A total of 130 patients were enrolled in the study. Median age of the overall study participants was 66, and all collected patient demographics were significantly different between the PAD and non-PAD groups except for sex and eGFR (Table 1). Among the 65 patients with PAD, there were 4 patients $(6 \%)$ with chronic limb threatening ischemia (CLTI) that had an average ABI of 0.54 and median age of 67 years. Relative to patients with PAD, the CLTI patients did not have any significant difference in measured demographic variables or cardiovascular risk factors. The uFABP3/uCr levels in the PAD group [median (IQR) 4.41 (2.79-8.08)] were 1.7 folds higher than levels of the non-PAD group [median (IQR) 2.49 (1.78-3.12)]. In additional analysis, we investigated the levels of the non-normalized uFABP3 in the PAD group. Our results demonstrated that the non-normalized uFABP3 


\begin{tabular}{|c|c|c|c|}
\hline Variable & Non-PAD $(n=65)$ & $\operatorname{PAD}(n=65)$ & p-value ${ }^{\alpha}$ \\
\hline \multicolumn{4}{|l|}{ Median $(\mathrm{IQR})^{\dagger}$} \\
\hline $\mathrm{ABI}$ & $1.06(1.00-1.13)$ & $0.52(0.45-0.69)$ & 0.001 \\
\hline Age in years & $56(43-68)$ & $72(65-80)$ & 0.001 \\
\hline eGFR & 88 (72.5-99.5) & $86(69.0-92.0)$ & 0.293 \\
\hline \multicolumn{4}{|l|}{ Frequency, $\mathbf{n}(\%)^{\ddagger}$} \\
\hline Sex-male & $36(55)$ & $45(69)$ & 0.103 \\
\hline Hypertension & $30(46)$ & $50(77)$ & 0.001 \\
\hline Hypercholesteremia & $23(35)$ & $49(75)$ & 0.001 \\
\hline Diabetes & $10(15)$ & $33(51)$ & 0.001 \\
\hline Smoking & $39(60)$ & $54(83)$ & 0.004 \\
\hline History of CAD & $7(11)$ & $28(44)$ & 0.001 \\
\hline History of stroke & $1(2)$ & $10(15)$ & 0.005 \\
\hline
\end{tabular}

Table 1. Patient demographics and clinical characteristics. Medians and interquartile ranges (IQR) were calculated for continuous variables. Frequencies and percentages were calculated for categorical variables; all numbers were rounded up with zero decimal place. All p-values were rounded to three decimal places. $A B I$ ankle brachial index, $e G F R$ estimated glomerular filtration rate. ${ }^{a}$ The significance of the difference between PAD and non-PAD groups. ${ }^{\dagger}$ Differences between groups were compared using Mann-Whitney test. ${ }^{\ddagger}$ Differences between groups were compared using chi-square test.

\begin{tabular}{|l|l|l|l|l|l|l|}
\hline \multirow{2}{*}{ Condition } & \multicolumn{2}{l}{ Non-PAD } & \multicolumn{2}{l|}{ PAD $^{9}$} \\
\cline { 2 - 7 } & Condition absent & Condition present & p-value $^{\dagger}$ & Condition absent & Condition present & p-value $^{\ddagger}$ \\
\hline Age $\geq 65$ & $2.44(1.76-3.33)$ & $2.60(2.33-2.93)$ & 0.820 & $3.69(3.11-8.64)$ & $4.77(2.62-8.08)$ & 0.915 \\
\hline Sex-male & $2.46(1.82-3.16)$ & $2.49(1.72-3.17)$ & 0.989 & $5.70(3.43-8.94)$ & $3.63(2.50-7.89)$ & 0.159 \\
\hline Hypertension & $2.24(1.64-2.83)$ & $2.70(2.28-3.67)$ & 0.067 & $3.90(2.80-6.77)$ & $4.45(2.76-8.78)$ & 0.383 \\
\hline Hypercholesteremia & $2.45(1.78-3.30)$ & $2.53(1.78-2.95)$ & 0.837 & $5.81(3.36-7.31)$ & $3.90(2.62-8.13)$ & 0.437 \\
\hline Diabetes & $2.45(1.77-3.21)$ & $2.65(2.14-3.43)$ & 0.513 & $3.81(2.85-6.85)$ & $5.87(2.74-8.13)$ & 0.609 \\
\hline Smoking & $2.43(1.72-3.66)$ & $2.50(1.80-3.04)$ & 0.893 & $4.77(3.13-9.08)$ & $4.07(2.76-8.08)$ & 0.391 \\
\hline History of CAD & $2.49(1.77-3.23)$ & $2.45(1.80-3.04)$ & 0.899 & $3.90(2.90-8.54)$ & $4.28(2.48-7.32)$ & 0.571 \\
\hline
\end{tabular}

Table 2. Median values of $\mathrm{uFABP} 3 / \mathrm{uCr}$ in $\mathrm{PAD}$ and non-PAD patients with and without various PAD risk factors and comorbidities. ${ }^{9}$ Median $\mathrm{uFABP} 3 / \mathrm{uCr}, \mu \mathrm{g} / \mathrm{g}$ (interquartile range) for each subgroup. ${ }^{\dagger}$ Difference among non-PAD patients. ${ }^{\ddagger}$ Difference among PAD patients.

in the PAD group [median (IQR) 3.48 (2.51-5.45), $\mu \mathrm{g} / \mathrm{g}$ ] were also significantly higher than in non-PAD controls [median (IQR) $1.89(0.93-3.40), \mu \mathrm{g} / \mathrm{g}$ ].

Correlation between uFABP3/uCr and PAD. To understand the association between $\mathrm{uFABP} 3 / \mathrm{uCr}$ and the measured cardiovascular risk factors, subgroup analysis were conducted. Our analysis demonstrated that risk factors had no significant effect on $\mathrm{UFABP} 3 / \mathrm{uCr}$ in both PAD and non-PAD patients (Table 2). We also investigated the association between $\mathrm{uFABP} 3 / \mathrm{uCr}$ and PAD severity (based on the ABI) using spearman test. Our test demonstrated a significant negative correlation between uFABP3/uCr and $A B I(\rho=-0.436$; $p$-value $=0.001$; supplemental Fig. 1). Next, we investigated levels of uFABP3/uCr after stratifying PAD patients based on their ABI as per the ESVM guidelines ${ }^{21}$. The stratified groups were: mild PAD (ABI 0.75-0.9, $\mathrm{n}=11$ ), moderate PAD $(A B I 0.74-0.50, n=29)$ and severe $\operatorname{PAD}(A B I<0.50, n=25)$. Our data shows a significant difference in median $\mathrm{uFABP} 3 / \mathrm{uCr}$ levels between the moderate and severe PAD subgroups when compared to the non-PAD group (p-value $<0.001$, for both) (Fig. 1). Although we noted a trend in increasing levels of uFABP3/uCr as the ABI worsens, no statistical difference was observed when comparing PAD subgroups specifically ( $\mathrm{p}$-value $=0.231$ ).

Influence of confounding factors on UFABP3/uCr levels in patients with PAD. To investigate the independent association between $\mathrm{uFABP} 3 / \mathrm{uCr}$ levels and PAD, multiple stepwise regression analysis was conducted. Our analysis demonstrated that $\mathrm{uFABP} 3 / \mathrm{uCr}$ levels were strongly associated with $\mathrm{PAD}$, even after adjusting for age and sex (model 1; OR, 2.02, 95\% confidence interval (95\% CI), 1.41-2.90, p-value =0.001). Table 3 displays the results of several other regression models that demonstrate a significant association between $\mathrm{uFABP} 3 / \mathrm{uCr}$ levels and PAD after adjusting for age, sex, prior history of CAD, smoking, eGFR and hypercholesteremia.

Diagnostic potential of UFABP3/uCr for PAD. Lastly, a ROC analysis was performed to measure the diagnostic accuracy of uFABP3/uCr in diagnosing patients with PAD. Prior to adjusting for confounding factors, 


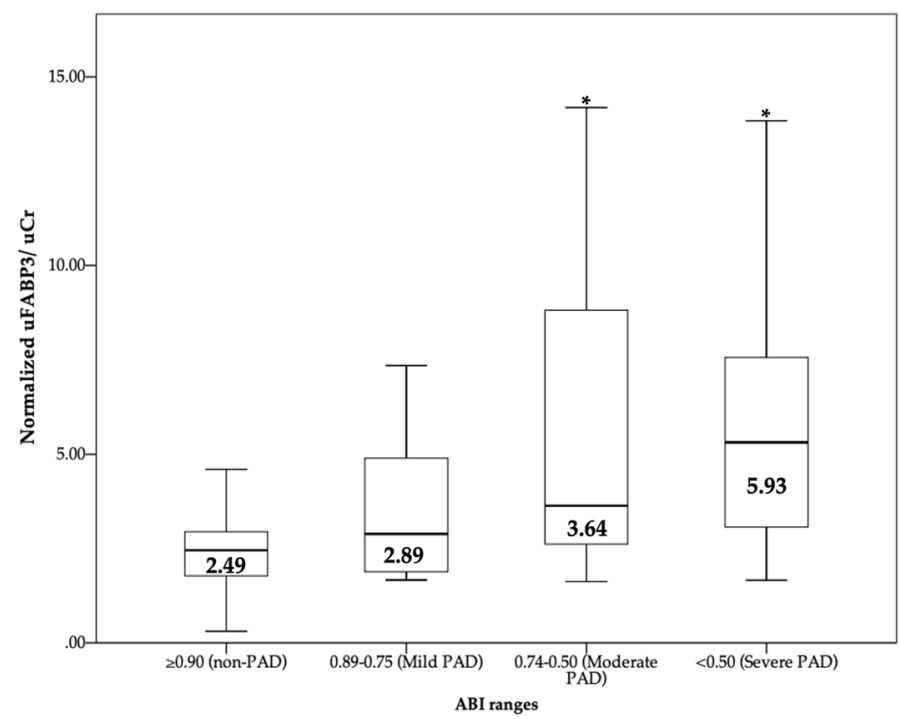

Figure 1. Boxplot illustrating the levels of normalized uFABP3/uCr among non-PAD controls $(\mathrm{n}=65)$ and PAD patients stratified based on their ABI values (mild PAD: 0.89-0.75, $\mathrm{n}=11$; moderate PAD: 0.74-0.50, $\mathrm{n}=29$; severe PAD: $<0.50, \mathrm{n}=25$ ). Significant difference in median uFABP3/uCr levels was noted between the moderate and severe PAD subgroups compared to non-PAD group, (p-value $<0.001$ ). Values displayed in figure represents median uFABP3/uCr levels values in each subgroup. ${ }^{*} \mathrm{p}$-value $<0.05$ compared to non-PAD group.

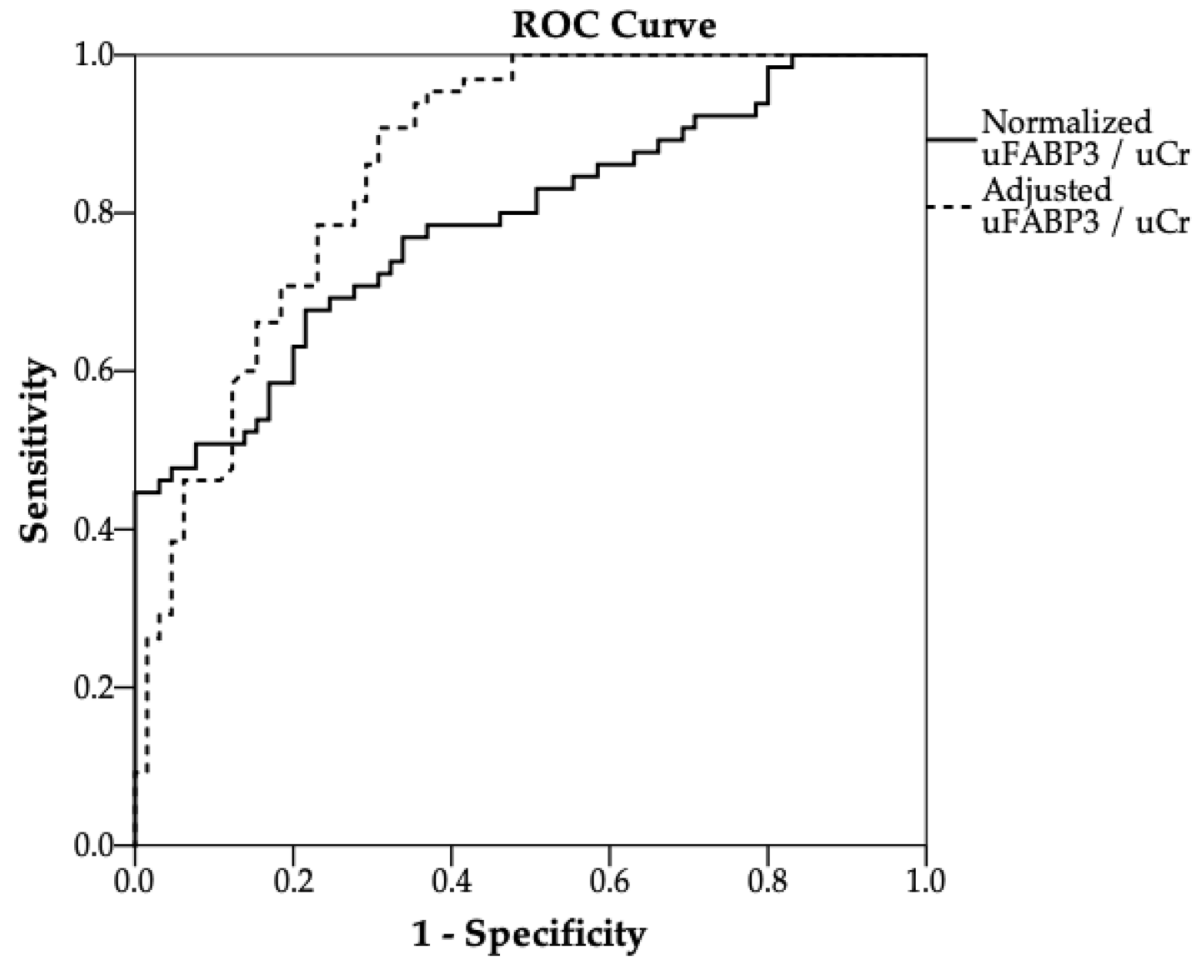

Figure 2. Receiver operating characteristic (ROC) curves of uFABP3/uCr for distinguishing patients with peripheral arterial disease (PAD, $n=65$ ) from patients without PAD (non-PAD, $n=65$ ) in an unadjusted model (solid line) and an adjusted model (dashed line). The area under the curve (AUC) and the 95\% confidence intervals for uFABP3/uCr improved from 0.79 (95\%: 0.71-0.87) to 0.86 (95\%: 0.80-0.92) after adjusting for eGFR, age, hypercholesteremia, smoking and diabetes.

the AUC for uFABP3/uCr was 0.79 (95\%: 0.71-0.87). After adjusting for age, eGFR, hypercholesteremia, smoking and diabetes, the ROC analysis for $\mathrm{uFABP} 3 / \mathrm{uCr}$ demonstrated an improved AUC of 0.86 (95\%: 0.80-0.92) (Fig. 2). 


\begin{tabular}{|l|l|l|}
\hline Regression models & Odds ratio $(\mathbf{9 5 \%}$ CI) & p-value \\
\hline Unadjusted model & $1.93(1.45-2.56)$ & 0.001 \\
\hline Model 1 (adjusted for age and sex) & $2.02(1.41-2.90)$ & 0.001 \\
\hline Model 1+CAD & $2.03(1.38-2.97)$ & 0.001 \\
\hline Model 1+CAD + smoking & $2.11(1.40-3.19)$ & 0.001 \\
\hline Model 1+CAD + smoking +hypercholesteremia & $2.15(1.39-3.34)$ & 0.001 \\
\hline Model 1+CAD + smoking +hypercholesteremia + eGFR & $2.34(1.47-3.75)$ & 0.001 \\
\hline
\end{tabular}

Table 3. Influence of individual factors on the odds ratios for PAD per one unit increase in normalized urinary fatty acid-binding protein 3 . CAD coronary arterial disease, $e G F R$ estimated glomerular filtration rate, CI confidence interval. ${ }^{*}$ Binary logistic regression models for PAD per one unit increase in uFABP3/uCr.

\section{Discussion}

In this study, an association was demonstrated between PAD and uFABP3/uCr. Our data shows uFABP3/uCr is elevated in patients with PAD when compared to non-PAD controls, even after adjusting for potential confounding factors. Furthermore, the ROC analysis suggests potential for $\mathrm{uFABP} 3 / \mathrm{uCr}$ in distinguishing PAD patients from non-PAD patients. Collectively, this data serves as a strong foundation for future studies investigating urine FABP3 as a biomarker for PAD.

Normally, biomarkers are used along with a clinical exam and radiographical investigations to enhance disease diagnosis. For instance, a diagnosis of acute myocardial infarction is reached after using a combination of several biomarkers (creatine kinase and troponin) in addition to an electrocardiogram and clinical symptoms. With regards to PAD, our research group previously identified that pFABP3 is potentially associated with PAD ${ }^{12}$, and through this study, have been able to demonstrate that uFABP3/uCr can serve as a potential diagnostic biomarker for PAD as well.

Fatty acid binding proteins (FABPs) play an important role in the trafficking of intracellular fatty acids ${ }^{13,22}$. Several studies have demonstrated the utility of uFABPs as potential biomarkers for diseased states. For instance, urinary FABP1 levels were noted to be higher in patients with septic shock when compared to patients with severe sepsis but without shock ${ }^{23}$. Tanaka et al. demonstrated that uFABP3 is elevated in patients with acute myocardial infraction ${ }^{24}$. Similarly, Nayashida et al. studied the influence of renal function on uFABP3 levels in patients undergoing a primary coronary artery bypass, and suggested, alongside others, that uFABP3 may be an early and sensitive biochemical marker for the diagnosis of myocardial injury in patients undergoing cardiac surgery ${ }^{15,25}$. Studies have also suggested that after myocardial injury, FABP3 filtration by the kidney can be impaired when creatinine clearance is decreased, resulting in lower levels of uFABP3 ${ }^{25,26}$. Thus, UFABP3 may be utilized as a potential diagnostic biomarker for PAD in the absence of reduced creatinine clearance. Urinary FABPs may also serve as potential biomarkers for the earlier diagnosis of acute renal failure ${ }^{27,28}$.

The present study is not without limitations. First, there was a relatively small sample size. Second, due to the small sample size, we were not able to adjust for all potential confounding factors. Thirdly, the cross-section study design of this pilot trial did not allow for patient follow-up. Lastly, patients with acute myocardial infarction and renal failure were not included due to their confounding effect on uFABP3.

\section{Conclusions}

In summary, our study revealed for the first time, that $\mathrm{uFABP} 3 / \mathrm{uCr}$ is significantly elevated in patients with PAD. Our data demonstrated an independent association between UFABP3 and PAD, and ROC analysis demonstrated strong ability to discriminate, raising its potential as a tool for PAD diagnosis. Therefore, uFABP3 is a potential diagnostic biomarker for PAD; however, larger clinical trials are needed to confirm these findings.

Received: 15 December 2020; Accepted: 10 May 2021

Published online: 26 May 2021

\section{References}

1. Conte, S. M. \& Vale, P. R. Peripheral arterial disease. Heart Lung Circ. 27, 427-432 (2018).

2. Fowkes, F. G. R. et al. Comparison of global estimates of prevalence and risk factors for peripheral artery disease in 2000 and 2010 : A systematic review and analysis. Lancet 382, 1329-1340 (2013).

3. Cornejo Del Rio, V. et al. Prevalence of peripheral artery disease (PAD) and factors associated: An epidemiological analysis from the population-based Screening PRE-diabetes and type 2 DIAbetes (SPREDIA-2) study. PLoS One 12, e0186220 (2017).

4. McDermott, M. M. et al. Asymptomatic peripheral arterial disease is associated with more adverse lower extremity characteristics than intermittent claudication. Circulation 117, 2484-2491. https://doi.org/10.1161/circulationaha.107.736108 (2008).

5. Sampson, U. K. et al. Global and regional burden of death and disability from peripheral artery disease: 21 world regions, 1990 to 2010. Global Heart 9, 145-158.e121 (2014).

6. Roth, G. A. et al. Trends and patterns of geographic variation in cardiovascular mortality among US counties, 1980-2014. JAMA 317, 1976-1992 (2017).

7. Subherwal, S. et al. Missed opportunities: Despite improvement in use of cardioprotective medications among patients with lowerextremity peripheral artery disease, underuse remains. Circulation 126, 1345-1354 (2012).

8. Aboyans, V., Ricco, J. \& Bartelink, M. Bjö rck M, Brodmann M, Cohnert T, et al. 2017 ESC Guidelines on the Diagnosis and Treatment of Peripheral Arterial Diseases, in collaboration with the European Society for Vascular Surgery (ESVS): Document covering atherosclerotic disease of extracranial carotid and vertebral, mesenteric, renal, upper and lower extremity arteries Endorsed by: 
the European Stroke Organization (ESO) The Task Force for the Diagnosis and Treatment of Peripheral Arterial Diseases of the European Society of Cardiology (ESC) and of the European Society for Vascular Surgery (ESVS). Eur. Heart J. 39, 763-816 (2018).

9. Nicolaï, S. P. et al. Ankle brachial index measurement in primary care: Are we doing it right?. Br. J. Gen. Pract. 59, 422-427 (2009).

10. Association, A. D. Peripheral arterial disease in people with diabetes. Diabetes Care 26, 3333-3341 (2003).

11. Conte, M. S. et al. Society for Vascular Surgery practice guidelines for atherosclerotic occlusive disease of the lower extremities: management of asymptomatic disease and claudication. J. Vasc. Surg. 61, 2S-41S.e41 (2015).

12. Syed, M. H. et al. Fatty acid binding protein 3 is associated with peripheral arterial disease. JVS Vasc. Sci. (2020).

13. Colli, A., Josa, M., Pomar, J. L., Mestres, C. A. \& Gherli, T. Heart fatty acid binding protein in the diagnosis of myocardial infarction: Where do we stand today?. Cardiology 108, 4-10. https://doi.org/10.1159/000095594 (2007).

14. Knowlton, A. A., Apstein, C. S., Saouf, R. \& Brecher, P. Leakage of heart fatty acid binding protein with ischemia and reperfusion in the rat. J. Mol. Cell. Cardiol. 21, 577-583. https://doi.org/10.1016/0022-2828(89)90823-7 (1989).

15. Nayashida, N. et al. Influence of renal function on serum and urinary heart fatty acid-binding protein levels. J. Cardiovasc. Surg. 42,735-740 (2001).

16. Al-Hadi, H. A., William, B. \& Fox, K. A. Serum level of heart-type fatty acid-binding protein in patients with chronic renal failure. Sultan Qaboos Univ. Med. J. 9, 311-314 (2009).

17. Furuhashi, M. et al. Serum fatty acid-binding protein 4 is a predictor of cardiovascular events in end-stage renal disease. PLoS ONE 6, e27356. https://doi.org/10.1371/journal.pone.0027356 (2011).

18. Zamzam, A. et al. Fatty acid binding protein 4-A circulating protein associated with peripheral arterial disease in diabetic patients. J. Clin. Med. 9. https://doi.org/10.3390/jcm9092843 (2020).

19. Syed, M. H. et al. MicroRNA profile of patients with chronic limb-threatening ischemia. Diagnostics (Basel, Switzerland) 10. https:// doi.org/10.3390/diagnostics10040230 (2020).

20. Levey, A. S. et al. A more accurate method to estimate glomerular filtration rate from serum creatinine: A new prediction equation. Ann. Intern. Med. 130, 461-470 (1999).

21. Frank, U. et al. ESVM guideline on peripheral arterial disease. Vasa (2019).

22. Nguyen, H. C., Qadura, M. \& Singh, K. K. Role of the fatty acid binding proteins in cardiovascular diseases: A systematic review. J. Clin. Med. 9. https://doi.org/10.3390/jcm9113390 (2020).

23. Nakamura, T., Sugaya, T. \& Koide, H. Urinary liver-type fatty acid-binding protein in septic shock: Effect of polymyxin B-immobilized fiber hemoperfusion. Shock (Augusta, Ga.) 31, 454-459. https://doi.org/10.1097/SHK.0b013e3181891131 (2009).

24. Tanaka, T., Hirota, Y., Sohmiya, K., Nishimura, S. \& Kawamura, K. Serum and urinary human heart fatty acid-binding protein in acute myocardial infarction. Clin. Biochem. 24, 195-201. https://doi.org/10.1016/0009-9120(91)90571-u (1991).

25. Hayashida, N. et al. Plasma and urinary levels of heart fatty acid-binding protein in patients undergoing cardiac surgery. Jpn. Circ. J. 64, 18-22. https://doi.org/10.1253/jcj.64.18 (2000).

26. Hofstra, J. M., Deegens, J. K., Steenbergen, E. J. \& Wetzels, J. F. Urinary excretion of fatty acid-binding proteins in idiopathic membranous nephropathy. Nephrol. Dial. Transplant. 23, 3160-3165. https://doi.org/10.1093/ndt/gfn190 (2008).

27. Noiri, E. et al. Urinary fatty acid-binding protein 1: An early predictive biomarker of kidney injury. Am. J. Physiol. Renal Physiol. 296, F669-679. https://doi.org/10.1152/ajprenal.90513.2008 (2009).

28. Doi, K. et al. Urinary L-type fatty acid-binding protein as a new biomarker of sepsis complicated with acute kidney injury. Crit. Care Med. 38, 2037-2042. https://doi.org/10.1097/CCM.0b013e3181eedac0 (2010).

\title{
Author contributions
}

Conceptualization-R.A., M.Q.; methodology-A.Z., R.A., M.Q.; formal analysis-A.Z., R.A., M.Q.; data interpretation-A.Z., M.H.S., J.H., J.E., K.K.S., R.A., M.Q.; writing and revision-A.Z., M.H.S., J.H., J.E., K.K.S., R.A., M.Q. All authors have read and agreed to the published version of the manuscript.

\section{Funding}

This research was funded by the Blair Foundation.

\section{Competing interests}

Dr. John Eikelboom reports consulting fees/honoraria and/or grant support from Astra-Zeneca, Bayer Boehringer-Ingelheim, Bristol-Myer-Squibb/Pfizer, Daiichi-Sankyo, Eli-Lilly, Glaxo-Smith-Kline, Pfizer, Janssen, Sanofi-Aventis, Servier. The funders also had no role in the design of the study; in the collection, analyses, or interpretation of data; in the writing of the manuscript, or in the decision to publish the results. All other authors declare no competing interests.

\section{Additional information}

Supplementary Information The online version contains supplementary material available at https://doi.org/ 10.1038/s41598-021-90395-0.

Correspondence and requests for materials should be addressed to M.Q.

Reprints and permissions information is available at www.nature.com/reprints.

Publisher's note Springer Nature remains neutral with regard to jurisdictional claims in published maps and institutional affiliations.

\begin{abstract}
Open Access This article is licensed under a Creative Commons Attribution 4.0 International License, which permits use, sharing, adaptation, distribution and reproduction in any medium or format, as long as you give appropriate credit to the original author(s) and the source, provide a link to the Creative Commons licence, and indicate if changes were made. The images or other third party material in this article are included in the article's Creative Commons licence, unless indicated otherwise in a credit line to the material. If material is not included in the article's Creative Commons licence and your intended use is not permitted by statutory regulation or exceeds the permitted use, you will need to obtain permission directly from the copyright holder. To view a copy of this licence, visit http://creativecommons.org/licenses/by/4.0/.
\end{abstract}

(C) The Author(s) 2021 